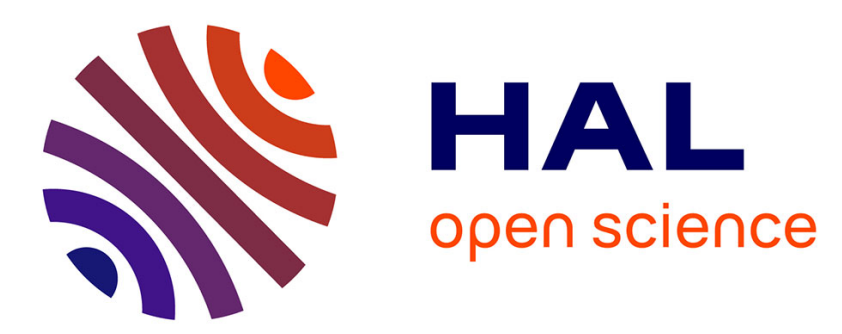

\title{
Role for membrane fluidity in ethanol-induced oxidative stress of primary rat hepatocytes.
}

Odile Sergent, Manuella Pereira, Corinne Belhomme, Martine Chevanne, Laurence Huc, Dominique Lagadic-Gossmann

\section{- To cite this version:}

Odile Sergent, Manuella Pereira, Corinne Belhomme, Martine Chevanne, Laurence Huc, et al.. Role for membrane fluidity in ethanol-induced oxidative stress of primary rat hepatocytes.. Journal of Pharmacology and Experimental Therapeutics, 2005, 313 (1), pp.104-11. 10.1124/jpet.104.078634 . hal-02677113

\section{HAL Id: hal-02677113 \\ https://hal.inrae.fr/hal-02677113}

Submitted on 31 May 2020

HAL is a multi-disciplinary open access archive for the deposit and dissemination of scientific research documents, whether they are published or not. The documents may come from teaching and research institutions in France or abroad, or from public or private research centers.
L'archive ouverte pluridisciplinaire HAL, est destinée au dépôt et à la diffusion de documents scientifiques de niveau recherche, publiés ou non, émanant des établissements d'enseignement et de recherche français ou étrangers, des laboratoires publics ou privés. 


\title{
Role for Membrane Fluidity in Ethanol-Induced Oxidative Stress of Primary Rat Hepatocytes
}

\author{
Odile Sergent, Manuella Pereira, Corinne Belhomme, Martine Chevanne, Laurence Huc, \\ and Dominique Lagadic-Gossmann
}

Groupe de Recherche en Thérapeutique Anticancéreuse, Unité Propre de Recherche et de l'Enseignement Supérieur 6027 (O.S., M.P., C.B., M.C.), and Institut National de la Santé et de la Recherche Médicale U620, Université de Rennes 1 (L.H., D.L.-G.), Rennes Cedex, France

Received October 1, 2004; accepted December 22, 2004

\begin{abstract}
The relationship between bulk membrane fluidizing effect of ethanol and its toxicity due to oxidative stress is still unknown. To elucidate this issue, membrane fluidity of primary rat hepatocytes was studied by measuring order parameter after inhibition of ethanol-induced oxidative stress. We showed that pretreating cells with either 4-methyl-pyrazole (to inhibit ethanol metabolism), thiourea [a reactive oxygen species (ROS) scavenger], or vitamin $\mathrm{E}$ (a free radical chain-breaking antioxidant) prevented the ethanol-induced increase in membrane fluidity, thus suggesting that ethanol metabolism and ROS formation were involved in this elevation. The effects of membrane stabilizing agents (ursodeoxycholic acid or ganglioside GM1), shown to prevent fluidification, next pointed to a role for this increase in membrane fluidity in the development of ethanol-
\end{abstract}

induced oxidative stress. Indeed, ROS production, lipid peroxidation, and cell death were all inhibited by these agents. In contrast, the fluidizing compounds Tween 20 or 2-(2methoxyethoxy) ethyl 8-(cis-2-n-octylcyclopropyl) octanoate, which increased the membrane fluidizing effect of ethanol, enhanced the related oxidative stress. Using electron paramagnetic resonance to determine low molecular weight iron, we finally demonstrated that membrane fluidity influence proceeded through an increase in low molecular weight iron to enhance oxidative stress. In conclusion, the present findings clearly highlight the pivotal role of membrane fluidity in ethanolinduced oxidative stress and the potential therapeutic effect of membrane stabilizing compounds.
Oxidative damage to lipids, proteins, and DNA after ethanol intoxication of the liver are very well described (Nordmann et al., 1992; Bailey and Cunningham, 2002). Since oxidative stress generated in hepatocytes is involved in processes as varied as ethanol-induced apoptosis (Kurose et al., 1997; Minama et al., 2002), immune reactions toward the liver (Albano, 2002), and activation of stellate cells and hence fibrogenesis (Nieto et al., 2002), this phenomenon seems to contribute to the pathogenesis of alcoholic liver diseases. Consequently, this emphasizes the necessity to fully characterize the molecular mechanisms of ethanol-induced oxidative stress in hepatocytes, especially during the early stages of the disease. It can result from ethanol metabolism in hepatocytes that triggers reactive oxygen species (ROS) pro-

This study was supported by Institut de Recherches Scientifiques sur les Boissons Grant 2002/12.

Article, publication date, and citation information can be found at http://jpet.aspetjournals.org.

doi:10.1124/jpet.104.078634. duction during both acute and chronic alcoholism (Sergent et al., 1995; Bailey and Cunningham, 1998). At the very early stage of the disease, changes in membrane fluidity could also play a role, because an increase in plasma membrane fluidity has been described after acute ethanol intoxication of WRL-68 hepatic cell lines (Gutierrez-Ruiz et al., 1995) or of primary rat hepatocytes (Benedetti et al., 1994). What is particularly interesting is that liver is the only organ to maintain this increase in membrane fluidity during chronic intoxication (Yamada and Lieber, 1984; Polokoff et al., 1985), even though chronic ethanol exposure of animals or liver cells would make membranes more resistant to the disordering effects of ethanol in vitro (Polokoff et al., 1985; GutierrezRuiz et al., 1995). However, to our knowledge, no study has looked for a close association between ROS production and the increase in liver membrane fluidity due to ethanol intoxication. Actually, the main findings have focused on mitochondrial inner membrane and have highlighted a role for a decreased fluidity in the impairment of the mitochondrial

ABBREVIATIONS: ROS, reactive oxygen species; GM1, monosialoganglioside $1 ; \mathrm{H}_{2} \mathrm{FDA}$, dihydrofluorescein diacetate; UDCA, sodium ursodeoxycholate; 12-DSA, 12-doxyl stearic acid; DMPO, 5,5-dimethyl-1-pyrroline $N$-oxide; $\mathrm{A}_{2} \mathrm{C}, 2$-(2-methoxyethoxy) ethyl 8-(cis-2-n-octylcyclopropyl) octanoate; EPR, electron paramagnetic resonance. 
transport of reduced glutathione, one of the most important antioxidants in cells (Lluis et al., 2003).

In the present study, we have performed a series of experiments in which bulk membrane fluidity was manipulated by incorporating membrane stabilizing agents or membrane fluidizing compounds in membranes of rat hepatocytes. This has allowed assessment of the effect of membrane fluidity changes on ethanol-induced oxidative stress. In addition, membrane fluidity was also measured after inhibition of ethanol-induced oxidative stress to determine whether an early oxidative stress can influence the physical state of hepatocyte membranes.

\section{Materials and Methods}

Chemicals. Minimum Eagle's medium and medium 199 with Hanks' salts were purchased from Biomedia (Boussens, France). Fetal calf serum was from Biowest (Nuaille, France). Liberase was from Roche Diagnostics (Meylan, France). Ethanol and thiourea were obtained from Prolabo (Paris, France). Monosialoganglioside 1 sodium salt (GM1) was from Alexis (Coger, Paris, France). Dihydrofluorescein diacetate $\left(\mathrm{H}_{2} \mathrm{FDA}\right)$ was provided by Molecular Probes (Interchim, Montluçon, France). Ursodeoxycholate sodium salt (UDCA) was from Calbiochem (Meudon, France). 12-Doxyl stearic acid (12-DSA), Hoechst 33342, $\alpha$-tocopherol, desferrioxamine, 5,5dimethyl-1-pyrroline $N$-oxide (DMPO), 4-methylpyrazole, xanthine, xanthine oxidase, polyoxyethylene sorbitan monolaurate (Tween 20), and 2-(2-methoxyethoxy) ethyl 8-(cis-2- $n$-octylcyclopropyl) octanoate $\left(\mathrm{A}_{2} \mathrm{C}\right)$ were all purchased from Sigma-Aldrich (Saint Quentin Fallavier, France).

Cell Isolation and Culture. Briefly, adult rat hepatocytes were isolated from 2-month-old Sprague-Dawley animals by perfusion of the liver as described previously, except that a $17 \mu \mathrm{g} / \mathrm{ml}$ liberase solution was used for dissociation of liver parenchymal cells instead of a collagenase solution (Guguen-Guillouzo et al., 1983). Routinely, more than $90 \%$ of the freshly isolated hepatocytes excluded trypan blue, and 500 million of hepatocytes could be obtained from a single rat liver perfusion. Cells obtained using this protocol were shown to synthesize albumin, a specific function of hepatocytes, and to form bile canalicular-like elements (Guguen-Guillouzo et al., 1983). Cells were seeded at densities of $30 \times 10^{3}$ cells/well in 96-well Falcon microplates (ROS evaluation), $3 \times 10^{6}$ cells in $25-\mathrm{cm}^{2}$ Falcon flasks (lipid peroxidation evaluation), $10 \times 10^{6}$ cells in $75-\mathrm{cm}^{2}$ Falcon flasks (membrane fluidity determination), and $20 \times 10^{6}$ cells in $175-\mathrm{cm}^{2}$ Falcon flasks (low molecular weight iron measurement) and cultured in a medium composed of $75 \%$ minimum Eagle's medium and 25\% medium 199 with Hanks' salts, supplemented with $10 \%$ fetal calf serum and containing $50 \mu \mathrm{g}$ of streptomycin, $5 \mu \mathrm{g}$ of penicillin, $5 \mu \mathrm{g}$ of bovine insulin, $1 \mathrm{mg}$ of bovine serum albumin, and $2.2 \mathrm{mg}$ of $\mathrm{NaHCO}_{3}$ per milliliter. The cells were kept at $37^{\circ} \mathrm{C}$ in an atmosphere of $5 \% \mathrm{CO}_{2}$ and $95 \%$ air. The medium was changed $3 \mathrm{~h}$ after seeding and substituted with the same medium as described above but deprived of serum. Culture treatments were in all cases started the day after seeding.

Culture Treatments. Ethanol at a final concentration of $50 \mathrm{mM}$ was added to rat hepatocytes and cultures maintained at $37^{\circ} \mathrm{C}$ for 0.5 to $24 \mathrm{~h}$. It should be noted that over our test ethanol exposure period, no significant evaporation could be found in culture media without cells (Sergent et al., 1995). In one set of experiments, some cultures were preincubated either for $1 \mathrm{~h}$ with membrane stabilizing agents (100 $\mu \mathrm{M}$ UDCA or $60 \mu \mathrm{M}$ GM1) or for $0.5 \mathrm{~h}$ with membrane fluidizers $\left(600 \mu \mathrm{g} / \mathrm{ml}\right.$ Tween 20 or $5 \mu \mathrm{M} \mathrm{A} \mathrm{A}_{2} \mathrm{C}$ ), and then the medium was discarded before adding ethanol. In the experiments seeking the mechanisms underlying the fluidizing effect of ethanol, hepatocyte cultures were pretreated either for $12 \mathrm{~h}$ with $250 \mu \mathrm{M} \alpha$-tocopherol, a lipid peroxidation inhibitor, for $1 \mathrm{~h}$ with $100 \mathrm{mM}$ thiourea, a ROS scavenger, or for $0.5 \mathrm{~h}$ with $1 \mathrm{mM}$ 4-methylpyrazole, an ethanol metabolism inhibitor. These latter agents were kept in medium during the incubation with ethanol.

Measurement of Membrane Fluidity. The fluidity of hepatocyte bulk membranes (i.e., plasma membrane and possibly endosome and lysosome membranes) was determined by a spin-labeling method using electron paramagnetic resonance (EPR), as described previously in other cell cultures (Ogura et al., 1988). At the end of each incubation period, the lipid bilayer of hepatocyte membranes were spin labeled by incubation, for $15 \mathrm{~min}$ at $37^{\circ} \mathrm{C}$, of hepatocyte suspensions with $50 \mu \mathrm{g} / \mathrm{ml} 12$-DSA, a fatty acid exhibiting a stable nitroxide radical ring at the C12-position. Cells were then washed three times with phosphate-buffered saline to remove the free spin label. The resultant pellet was then transferred to a disposable glass capillary. The EPR spectra of labeled samples were acquired at ambient temperature on a Bruker 106 EPR spectrometer operating at 3495-G center field, $20-\mathrm{mW}$ microwave power, $9.82-\mathrm{GHz}$ microwave frequency, $1.771-\mathrm{G}$ modulation amplitude, and $100-\mathrm{kHz}$ modulation frequency. The fluidity of the labeled membrane was quantified by calculating the order parameter $\mathrm{S}$ according to equations described previously (Ogura et al., 1988). An increase in the value of $\mathrm{S}$ is interpreted as a decrease in membrane fluidity, whereas a decrease of this parameter reflects an increase in membrane fluidity.

Determination of Reactive Oxygen Species Production. Intracellular levels of ROS were measured using the nonfluorescent probe, $\mathrm{H}_{2}$ FDA, as described previously (Hempel et al., 1999). Oxidation by ROS causes a conversion of this probe into fluorescent fluorescein. Briefly, hepatocytes were loaded with $50 \mu \mathrm{M} \mathrm{H_{2 }}$ FDA in HEPES-buffered solution for $30 \mathrm{~min}$ at $37^{\circ} \mathrm{C}$, washed with HEPES, and then treated with $50 \mathrm{mM}$ ethanol in usual culture medium. After a last wash, fluorescence was directly recorded by a SpectraMax Gemini spectrofluorimeter (Molecular Devices, Saint-Grégoire, France) using 485-nm excitation and 530-nm emission wavelengths.

Evaluation of Lipid Peroxidation. Lipid peroxidation was analyzed by measurement of extracellular free malondialdehyde as described previously (Morel et al., 1990). Briefly, malondialdehyde was measured by size exclusion chromatography after ultrafiltration of the culture media through 500-Da membrane (Millipore, SaintQuentin-les-Yvelines, France).

Measurement of Low Molecular Weight Iron. Measurement of intracellular low molecular weight iron was based upon the capacity of desferrioxamine to chelate only low molecular weight iron and to give a paramagnetic chelate, which had the advantage to be directly detectable by EPR in whole hepatocytes (Sergent et al., 1997a)

Cell Death Determination by Microscopy. Because neither caspase activity nor lactic dehydrogenase release could be detected under our experimental conditions (our unpublished results), cell death was studied by visualization of nuclear morphology (condensed and fragmented chromatin) and by estimation of lysosome disruption. Nuclear fragmentation was monitored by Hoechst 33342 staining (excitation, $330-380 \mathrm{~nm}$; emission, $460 \mathrm{~nm}$ ). After $5 \mathrm{~h}$ of incubation with ethanol, cells were stained in PBS with $2 \mathrm{mg} / \mathrm{ml}$ Hoechst dye for $15 \mathrm{~min}$ at $37^{\circ} \mathrm{C}$ in the dark. Cells were examined using an inverted Olympus microscope, and the percentage of cells with fragmented nuclei was calculated as (cells with fragmented nuclei/total cells $) \times 100$. Total population was always $>300$ cells.

Lysosome disruption was estimated from acridine orange-stained hepatocytes, knowing that an increase in green acridine orange fluorescence reflected such a disruption (Zhao et al., 2000). Briefly, cultures were incubated with $5 \mu \mathrm{M}$ acridine orange for $20 \mathrm{~min}$ at $37^{\circ} \mathrm{C}$ before being detached by incubation with trypsin/EDTA. After resuspension of cells in culture medium, cell suspensions were immediately analyzed by flow cytometry on an FACS Calibur flow cytometer (BD Biosciences, San Jose, CA). Fluorescence emission from acridine orange was detected at FL1 (green acridine orange fluorescence) after excitation at $488 \mathrm{~nm}$. Each measurement was conducted on 20,000 events and analyzed on Cell Quest software (BD Biosciences). 
Free Radical Scavenging Properties. Scavenging properties of membrane stabilizing agents toward hydroxyl radical $\left({ }^{\circ} \mathrm{OH}\right)$ and superoxide anion $\left(\mathrm{O}_{2}^{\overline{-}}\right)$ were examined by spin trapping using DMPO as a spin trap. For the ${ }^{\circ} \mathrm{OH}$ radical generation, the so-called Fenton reaction was used. Briefly, reaction mixture was constituted as follows: $30 \mu \mathrm{l}$ of distilled water, $50 \mu \mathrm{l}$ of $40 \mathrm{mM} \mathrm{H}_{2} \mathrm{O}_{2}, 10 \mu \mathrm{l}$ of $800 \mathrm{mM}$ DMPO, and $90 \mu \mathrm{l}$ of $1 \mathrm{mM}$ ferrous sulfate supplemented with either $20 \mu \mathrm{l}$ of distilled water or $20 \mu \mathrm{l}$ of $1 \mathrm{mM}$ UDCA, or $20 \mu \mathrm{l}$ of $600 \mu \mathrm{M}$ GM1. For the spin trapping experiments of the $\mathrm{O}_{2}^{-}$, the xanthine/ xanthine oxidase reaction was used to generate $\mathrm{O}_{2}{ }^{-}$by preparing the following reaction mixture: $30 \mu \mathrm{l}$ of $26 \mathrm{U} / \mathrm{ml}$ xanthine oxidase, $30 \mu \mathrm{l}$ of $5 \mathrm{mM}$ xanthine, $20 \mu \mathrm{l}$ of $400 \mathrm{mM}$ DMPO, and $100 \mu \mathrm{l}$ of ethanol supplemented with either $20 \mu \mathrm{l}$ of distilled water or $20 \mu \mathrm{l}$ of $1 \mathrm{mM}$ UDCA, or $20 \mu \mathrm{l}$ of $600 \mu \mathrm{M}$ GM1. Immediately after a vigorous stirring, the reaction mixtures were injected inside a glass capillary. For ${ }^{\circ} \mathrm{OH}$ or $\mathrm{O}_{2}{ }^{-}$detection, EPR spectra were recorded after 1 or 2 min, respectively, on a Bruker $106 \mathrm{EPR}$ spectrometer at room temperature with the EPR settings as follows: $3495-\mathrm{G}$ field center, 9.81-GHz microwave frequency, $20-\mathrm{mW}$ microwave power, $100-\mathrm{kHz}$ frequency modulation, 0.804-G modulation amplitude, 81.92-ms conversion time, and 1.28-ms time constant. The intensities of EPR spectra were represented by the double integration of the lines of lowest field. Free radical scavenging activities of UDCA and GM1 were expressed as percentage of decrease in the EPR intensity of the control performed with distilled water.

Statistical Analysis. Values were expressed as means \pm S.D. Hepatocytes obtained from three different isolations were generally used for all the protocols carried out. Multiple comparisons among the groups were performed using analysis of variance followed by the parametric Student-Newman-Keuls test or the nonparametric Mann-Whitney test, depending on the results of the normality test. Differences were considered significant at $p<0.05$.

\section{Results}

Early Increase in Membrane Fluidity upon Ethanol Exposure Involved Oxidative Stress. To investigate the kinetics of ethanol-induced membrane fluidizing effect, rat hepatocytes were treated with $50 \mathrm{mM}$ ethanol for different incubation times (Fig. 1A). The values were expressed as percentage of deviation from the order parameter calculated from untreated cells. An early increase $(+3.3 \%)$ in membrane fluidity of rat hepatocytes upon ethanol exposure was detectable after $30 \mathrm{~min}$ of incubation. Longer incubation times resulted in a more extensive rise, with a deviation percentage of $+8.7 \%$ at $5 \mathrm{~h}$. For the 24-h-incubation time, no change could be observed compared with control hepatocytes (data not shown). Because ethanol metabolism is well known to induce an oxidative stress that may affect membrane fluidity, the effect of ethanol on membrane fluidity was also evaluated after pretreatment of hepatocytes either by 4-methylpyrazole, an inhibitor of ethanol metabolism; by thiourea, an ROS scavenger; or by vitamin $\mathrm{E}$, a free radical chain-breaking antioxidant. It should be noted that 4-methyl-pyrazole, at this concentration, inhibited ethanol elimination by $95 \%$ (our unpublished results). As shown in Fig. 1B, all these compounds prevented the increase in membrane fluidity due to ethanol. Indeed, in 4-methyl-pyrazole-, thiourea-, or vitamin E-pretreated hepatocytes, no significant differences in the order parameter were found between ethanol-treated and untreated cultures.

The Membrane Fluidizing Effect of Ethanol Was Responsible for an Enhancement of Oxidative Stress. To determine whether the increase in membrane fluidity could participate in oxidative stress induced by ethanol, membrane
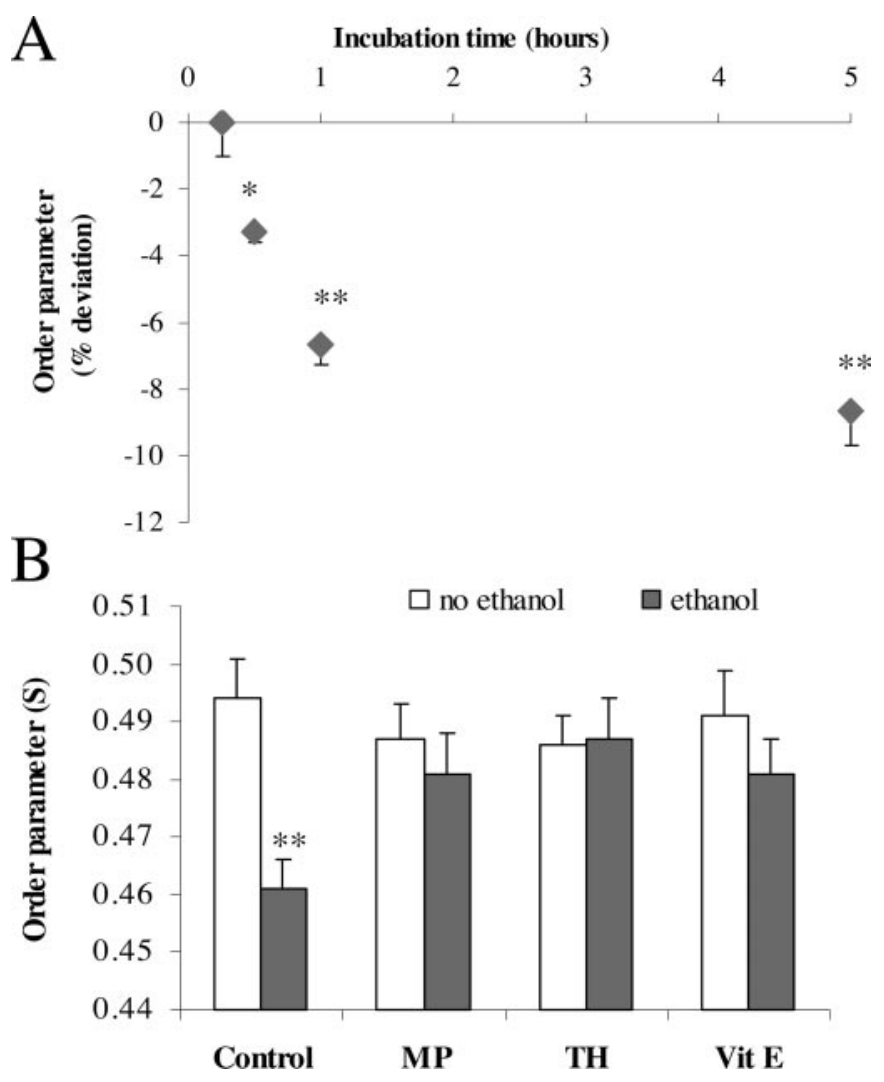

Fig. 1. Ethanol increased membrane fluidity in rat hepatocytes. A, changes in the membrane fluidity of hepatocytes treated with $50 \mathrm{mM}$ ethanol for $0.25,0.5,1,5$, or $24 \mathrm{~h}$. The membrane fluidity was monitored by EPR analysis of 12-DSA embedded in membranes. This analysis resulted in the obtention of the order parameter $\mathrm{S}$ that is inversely related to membrane fluidity. Data are expressed as a percentage of deviation from those obtained in untreated control cultures. B, effects of 4-methyl-pyrazole, an inhibitor of ethanol metabolism; thiourea, an ROS scavenger; and vitamin $\mathrm{E}$, a free radical chain-breaking antioxidant, on the increase of membrane fluidity in hepatocytes treated with $50 \mathrm{mM}$ ethanol for $1 \mathrm{~h}$. Some rat hepatocyte cultures were preincubated with 1 $\mathrm{mM}$ 4-methyl-pyrazole (4MP) for $0.5 \mathrm{~h}, 100 \mathrm{mM}$ thiourea (TH) for $1 \mathrm{~h}$, or with $250 \mu \mathrm{M}$ vitamin $\mathrm{E}$ (Vit E) for $12 \mathrm{~h}$. Results were obtained as in A, except that data were directly expressed as order parameter (S).Values are the mean \pm S.D. of three independent experiments. Ethanol-treated versus untreated cultures: $*, p<0.05 ; * *, p<0.01$.

stabilizing agents such as GM1 ganglioside or UDCA were added before addition of ethanol. These agents were first confirmed as being capable of blocking the membrane fluidizing effect of ethanol since, after pretreatment of cells with GM1 or UDCA, ethanol remained without effect on the order parameter (Fig. 2). In rat hepatocytes treated with ethanol, a rise in ROS production was detectable as soon as $15 \mathrm{~min}$ (Fig. $3 \mathrm{~A})$. After 1 and $5 \mathrm{~h}$ of incubation, the level of ROS was further increased, by nearly 2-fold, compared with untreated cells (Fig. 3, B and C, respectively); no significant effect of ethanol was detected at $24 \mathrm{~h}$ (data not shown). Interestingly, membrane stabilizing agents displayed no effect on ROS formation induced by a 15-min exposure to ethanol (Fig. 3A), whereas after 1 or $5 \mathrm{~h}$ of treatment, a significant protection against ROS production was observed (Fig. 3, B and C). Besides, we found that 4-methyl-pyrazole inhibited ROS formation as soon as 15 min (Fig. 3A). Note that ROS production increases in all groups over time. This is due to the primary culture senescence. Indeed, it is well known that many antioxidant enzymes, such as $\mathrm{Cu}, \mathrm{Zn}$ superoxide dismutase, glu- 


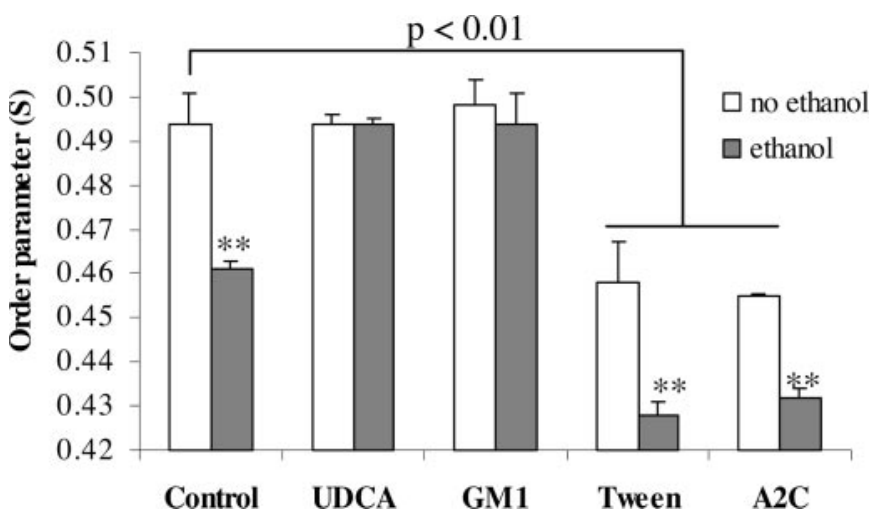

Fig. 2. Effects of membrane stabilizing agents (UDCA or GM1) or membrane fluidizers (Tween 20 or $\mathrm{A}_{2} \mathrm{C}$ ) on the membrane fluidizing effect of ethanol. The membrane fluidity was monitored by EPR analysis of 12DSA embedded in membranes. This analysis allowed the estimation of the order parameter $\mathrm{S}$ that is inversely related to membrane fluidity. Rat hepatocytes were incubated or not with $50 \mathrm{mM}$ ethanol for $1 \mathrm{~h}$. Some cultures were pretreated, for $1 \mathrm{~h}$, with membrane stabilizing agents (100 $\mu \mathrm{M}$ UDCA or $60 \mu \mathrm{M}$ GM1) or for $0.5 \mathrm{~h}$ with membrane fluidizers (600 $\mu \mathrm{g} / \mathrm{ml}$ Tween 20 or $\left.5 \mu \mathrm{g} / \mathrm{ml} \mathrm{A}_{2} \mathrm{C}\right)$. Values are the mean \pm S.D. of three independent experiments. Ethanol-treated versus untreated cultures: **, $p<0.01$.

tathione peroxidase, or catalase, are less expressed over incubation time after isolation of rat hepatocytes (Antras-Ferry et al., 1997).

To fully demonstrate the involvement of the increase in membrane fluidity in ethanol induced-oxidative stress, lipid peroxidation and also cell death were next followed, since oxidative stress generated by ethanol metabolism participates in ethanol-induced cell death (Kurose et al., 1997; Minama et al., 2002). Using malondialdehyde as a marker of lipid peroxidation, an increase by about $160 \%$ of lipid peroxidation was observed in hepatocyte cultures treated for $5 \mathrm{~h}$ with ethanol (Fig. 4A). This elevation of malondialdehyde content was inhibited by pretreating cells with membrane stabilizing agents GM1 or UDCA (Fig. 4A). In addition, both these agents prevented ethanol-induced cell death (Fig. 4, B and C). For lower concentrations of ethanol (10, 20, and 30 $\mathrm{mM}$ ), UDCA exhibited a similar protection on both ROS formation and lipid peroxidation (data not shown).

The involvement of the membrane fluidizing effect of ethanol in its capacity to generate oxidative stress was further confirmed by adding Tween 20, a mild neutral detergent, or $\mathrm{A}_{2} \mathrm{C}$, a fatty acid-like compound, to hepatocytes before ethanol treatment. Indeed, Tween 20 and $\mathrm{A}_{2} \mathrm{C}$ were demonstrated to increase membrane fluidity under our experimental conditions (Fig. 2), both in the presence or absence of ethanol. This elevation of membrane fluidity might be responsible for potentiation of ethanol-induced oxidative stress (Fig. 5, A and B) and cell death (Fig. 5C) in cultures pretreated by Tween 20 or $\mathrm{A}_{2} \mathrm{C}$. Note also that Tween 20 or $\mathrm{A}_{2} \mathrm{C}$ alone induced slight but significant effects on oxidative stress $(p<0.01)$. In addition, the supplementation of hepatocytes with UDCA, before exposure to Tween 20 or $\mathrm{A}_{2} \mathrm{C}$, protected cells from the potentiation by Tween 20 or $\mathrm{A}_{2} \mathrm{C}$ of ethanol-induced ROS formation (Fig. 5A). Similar effect was observed with GM1 (data not shown). It should be added that membrane stabilizing agents or fluidizing compounds did not change the rate of ethanol uptake by hepatocytes (data not shown).

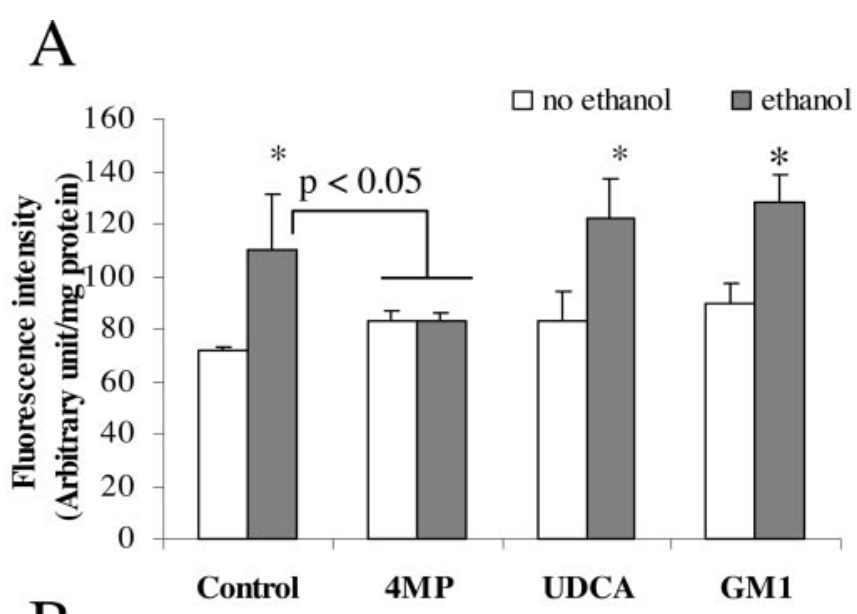

B
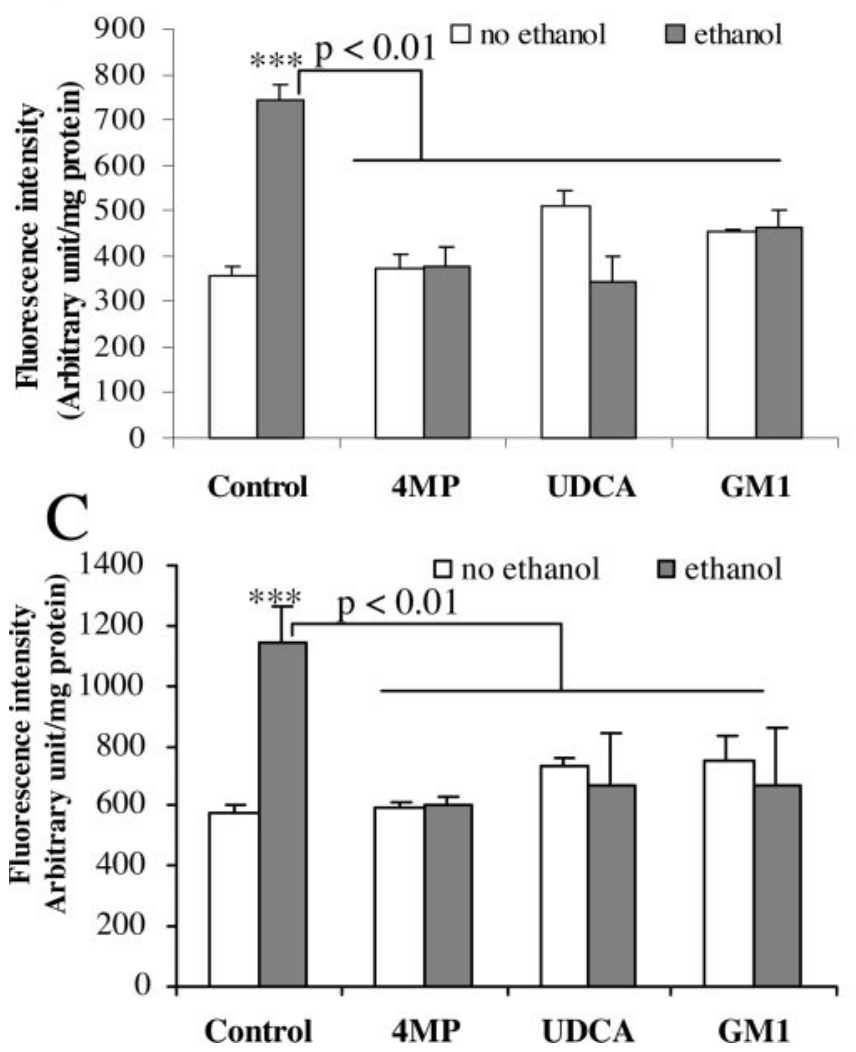

Fig. 3. Effects of membrane stabilizing agents (UDCA or GM1) or of 4-methyl-pyrazole, an inhibitor of ethanol metabolism, on ROS production induced by ethanol. ROS production was followed by the fluorescence of $\mathrm{H}_{2}$ FDA. Rat hepatocytes were incubated or not with $50 \mathrm{mM}$ ethanol for $15 \mathrm{~min}(\mathrm{~A}), 1 \mathrm{~h}$ (B), or $5 \mathrm{~h}$ (C). Some cultures were pretreated for $1 \mathrm{~h}$ with $100 \mu \mathrm{M}$ UDCA or $60 \mu \mathrm{M}$ GM1. Values are the mean \pm S.D. of three independent experiments. Ethanol-treated versus untreated cultures: *, $p<0.05 ; * * *, p<0.001$.

The Protection Afforded by Membrane Stabilizing Agents Was Not Due to a Direct Antioxidant Property. With the aim of seeking a possible direct antioxidant property of membrane stabilizing agents, which might have explained the above-mentioned results, UDCA and GM1, at the same concentrations as those used above, were tested by EPR for their capacity to scavenge superoxide anion or hydroxyl radical. UDCA and GM1 did not display a potent scavenging capacity (data not shown). Indeed, UDCA was the only agent to scavenge superoxide anion, 
A
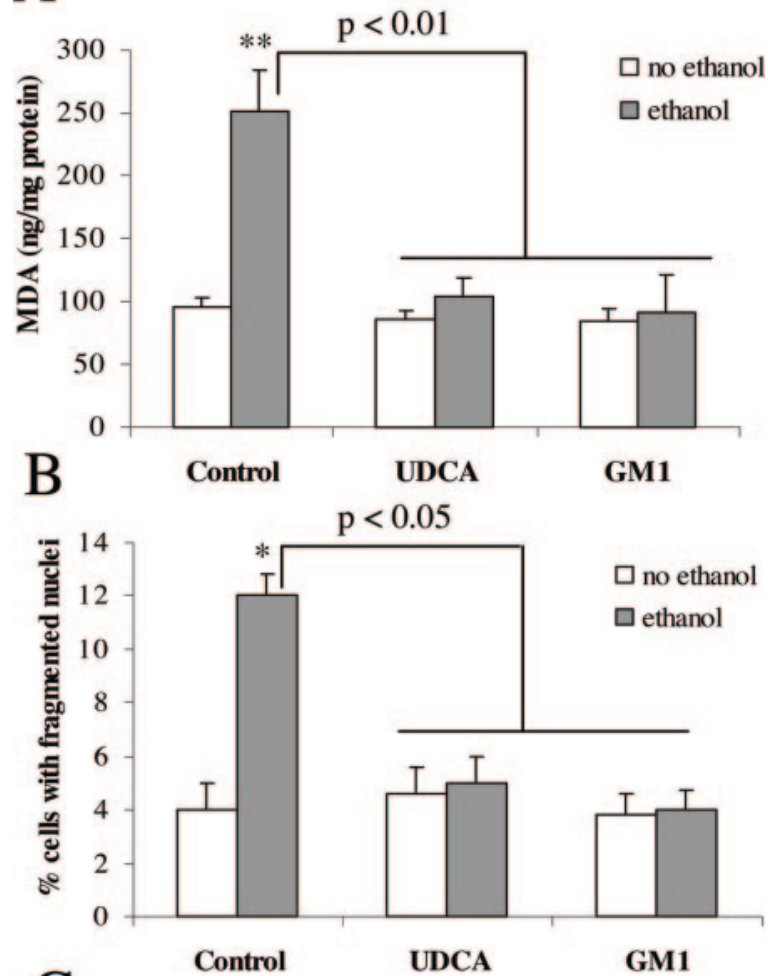

$\mathrm{C}$

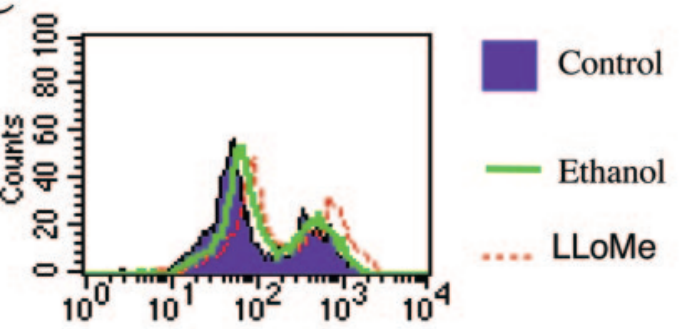

Green fluorescence

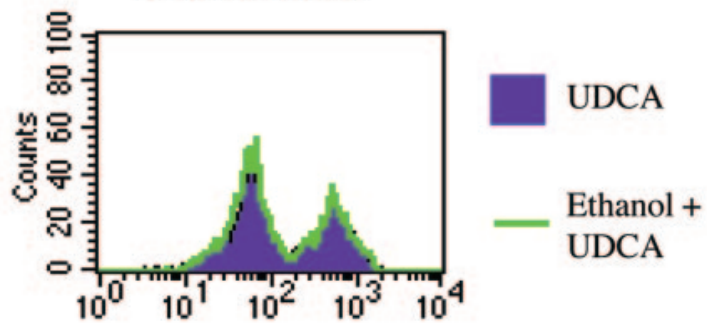

Green fluorescence

Fig. 4. Membrane stabilizing agents (UDCA or GM1) prevented ethanolinduced lipid peroxidation (A) and cell death (B and C). Lipid peroxidation was estimated by measurement of free malondialdehyde (MDA). Cell death was identified by staining nuclear chromatin with Hoechst 33342 (B) and or by staining lysosomes with acridine orange (C). L-leucyl-LLeucine methylester (LLoMe) $\left(500 \mu \mathrm{M}, 20 \mathrm{~min}\right.$ at $\left.37^{\circ} \mathrm{C}\right)$ was used as a positive control of lysosome disruption. Rat hepatocytes were incubated or not with $50 \mathrm{mM}$ ethanol for $5 \mathrm{~h}$. Some cultures were pretreated for $1 \mathrm{~h}$ with $100 \mu \mathrm{M}$ UDCA or $60 \mu \mathrm{M}$ GM1. Values are the mean \pm S.D. of three independent experiments, except for nuclear fragmentation experiments $(n=3$ or 5$)$. Ethanol-treated versus untreated cultures: $*, p<0.05$; **, $p<0.01$.

and this superoxide anion scavenging capacity was very weak $(21 \%)$.

To rule out the possibility of a direct antioxidant effect of UDCA or GM1, these drugs were also tested for their capac-
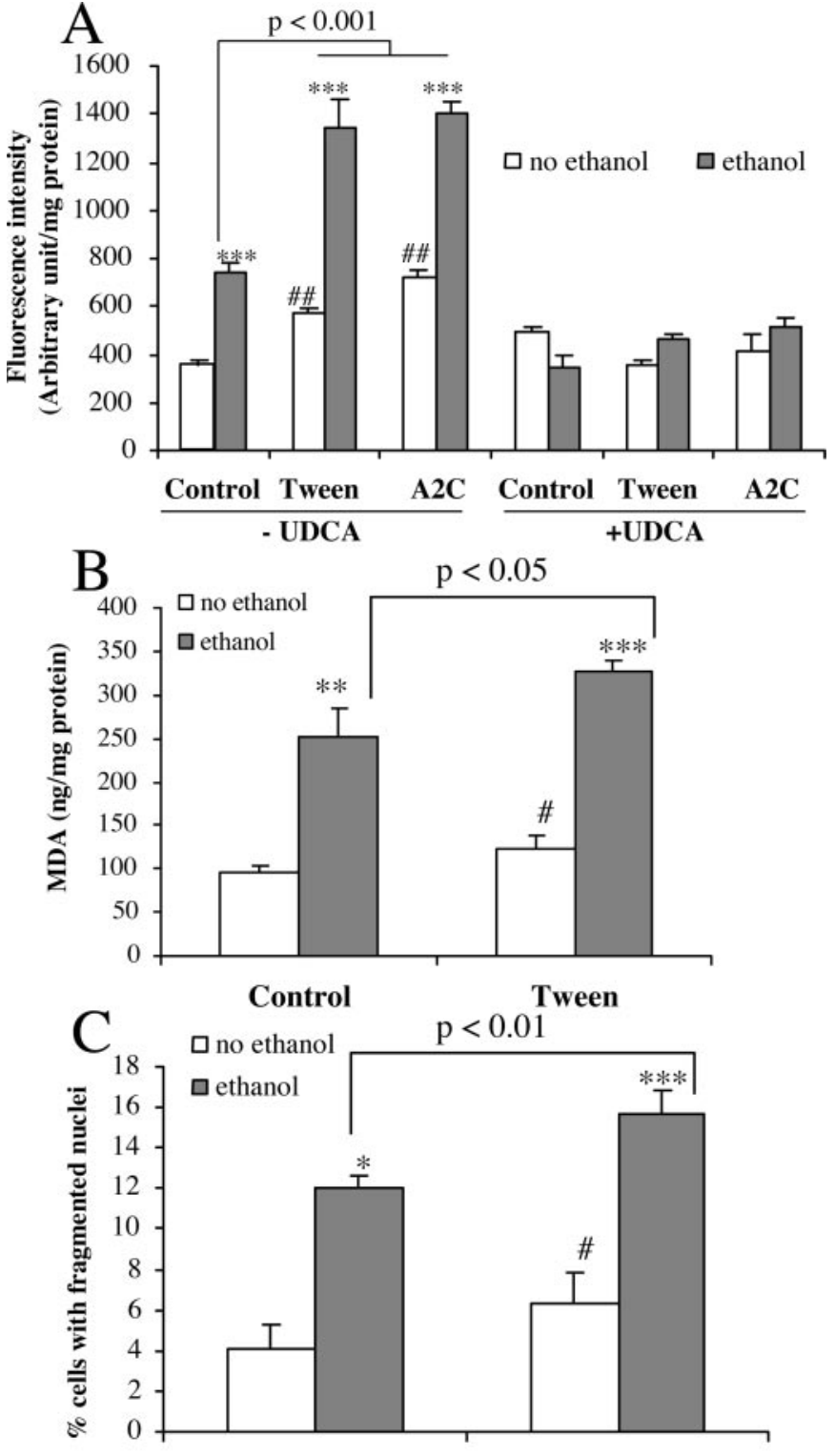

Control

Tween

Fig. 5. Effects of membrane fluidizers (Tween 20 or $\mathrm{A}_{2} \mathrm{C}$ ) on ROS formation (A), lipid peroxidation (B), and cell death (C) in rat hepatocytes incubated with ethanol. The membrane fluidity was monitored by EPR analysis of 12-DSA embedded in membranes. The estimated order parameter $\mathrm{S}$ is inversely related to membrane fluidity. ROS production was followed by the fluorescence of $\mathrm{H}_{2} \mathrm{FDA}$. Lipid peroxidation was estimated by measurement of MDA. Cell death was identified by staining nuclear chromatin with Hoechst 33342. Concerning studies on ROS formation, rat hepatocytes were incubated or not with $50 \mathrm{mM}$ ethanol for $1 \mathrm{~h}$. For studies on lipid peroxidation and cell death, ethanol was added for $5 \mathrm{~h}$. Some cultures were pretreated for $0.5 \mathrm{~h}$ with $600 \mu \mathrm{g} / \mathrm{ml}$ Tween 20 or 5 $\mu \mathrm{g} / \mathrm{ml} \mathrm{A}_{2} \mathrm{C}$. Values are the mean \pm S.D. of three independent experiments, except for cell death experiments $(n=3$ or 5$)$. Ethanol-treated versus untreated cultures: *, $p<0.05 ; * *, p<0.01$; ***, $p<0.001 . \mathrm{A}_{2} \mathrm{C}$ or Tween 20-treated versus untreated cultures: \#, $p<0.05$; \#\#, $p<0.01$.

ity to protect cells from lipid peroxidation induced by iron. This was carried out using a 5-h exposure to $20 \mu \mathrm{M}$ ferric nitriloacetate. Lipid peroxidation was followed by measuring malondialdehyde, a secondary end product of degradation of oxidized polyunsaturated fatty acids. UDCA and GM1 were unable to abolish the increase in malondialdehyde in hepatocytes treated by ferric nitriloacetate (control, $95 \pm 6 \mathrm{ng} / \mathrm{mg}$ protein; UDCA, $86 \pm 7 \mathrm{ng} / \mathrm{mg}$ protein; GM1, $79 \pm 4 \mathrm{ng} / \mathrm{mg}$ 
protein; iron, $1012 \pm 51 \mathrm{ng} / \mathrm{mg}$ protein; UDCA + iron, $944 \pm$ $130 \mathrm{ng} / \mathrm{mg}$ protein; and GM1 + iron, $915 \pm 87 \mathrm{ng} / \mathrm{mg}$ protein; $n=3$ independent experiments). Even with ferric nitriloacetate at a lower concentration $(5 \mu \mathrm{M})$, which led to a lesser extent of lipid peroxidation, UDCA and GM1 did not succeed to reduce malondialdehyde levels to those of the controls (data not shown). Together, these data strongly emphasized that membrane stabilizing agents, at the concentration used in our experiments, did not exhibit any direct antioxidant property.

The Involvement of Membrane Fluidity in EthanolInduced Oxidative Stress Proceeded through an Increase in Low Molecular Weight Iron Content. Low molecular weight iron consists of iron species that are not contained in high molecular weight molecules, such as ferritin or mitochondrial ferroproteins, but are able to trigger oxidative stress by catalyzing the formation of a highly reactive free radical, the hydroxyl radical, via Fenton or HaberWeiss reaction. An EPR technique was used to directly measure the level of low molecular weight iron in intact cells. As expected from previous studies (Sergent et al., 1995, 1997a), ethanol increased the content in low molecular weight iron by nearly $40 \%$ (Fig. 6A). UDCA and GM1 pretreatment totally
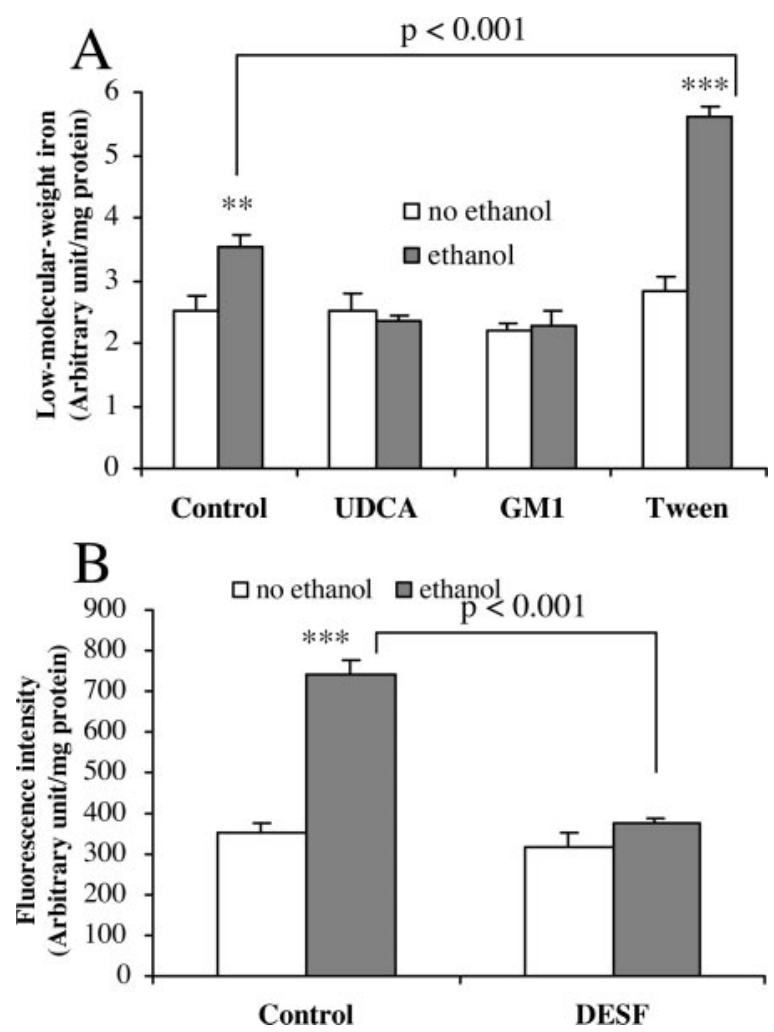

Fig. 6. Changes in low molecular weight iron content can account for the involvement of membrane fluidity in ethanol-induced oxidative stress. A effects of membrane stabilizing agents (UDCA and GM1) and Tween 20 on low molecular weight iron content in rat hepatocytes treated with ethanol for $1 \mathrm{~h}$. Low molecular weight iron was determined by EPR analysis after chelation by $1 \mathrm{mM}$ desferrioxamine. Rat hepatocytes were preincubated either for $1 \mathrm{~h}$ with $100 \mu \mathrm{M}$ UDCA or $60 \mu \mathrm{M}$ GM1 or for $0.5 \mathrm{~h}$ with $600 \mu \mathrm{g} / \mathrm{ml}$ Tween 20 . Values are the mean \pm S.D. of three independent experiments. Ethanol-treated versus untreated cultures: $* *, p<$ $0.01 ; * * *, p<0.001$. B, effect of desferrioxamine, a low molecular weight iron chelator, on ROS formation induced by ethanol. Rat hepatocytes were incubated with ethanol for $1 \mathrm{~h}$ or not. Some cultures were pretreated for $1 \mathrm{~h}$ with $20 \mu \mathrm{M}$ desferrioxamine (DESF). Ethanol-treated versus untreated cultures; ***, $p<0.001$. protected cells from this elevation, whereas Tween 20 pretreatment enhanced it (Fig. 6A). Because ROS formation could be inhibited by desferrioxamine, a low molecular weight iron chelator (Fig. 6B) and because ethanol-induced lipid peroxidation has been previously described to be inhibited by deferiprone, another low molecular weight iron chelator (Sergent et al., 1997b), it can be concluded from the present results that the membrane fluidizing effect of ethanol contributed to ethanol-induced oxidative stress by promoting the elevation of low molecular weight iron.

\section{Discussion}

The present study demonstrates, for the first time, that an increase in bulk membrane fluidity can amplify the oxidative stress induced by ethanol in the liver. In addition, we have found a new interpretation based upon an early ROS production to explain how ethanol can very rapidly increase membrane fluidity. Thus, in rat hepatocytes, in only $30 \mathrm{~min}$ of incubation, ethanol elicited an early membrane fluidizing effect. Whereas the fluidizing effect of ethanol had been ascribed until now to the disruption of the orderly packing between adjacent acyl chains due to either direct entering of ethanol into the lipid portion of the membrane (Rottenberg, 1991), an increased phosphatidylcholine/sphingomyelin ratio (Polokoff et al., 1985), or a decreased cholesterol/phospholipid ratio (Yamada and Lieber, 1984) of liver plasma membranes, we showed here that oxidative stress due to ethanol metabolism also contributed to the elevation of membrane fluidity since this rise was prevented by both antioxidants and 4-methyl-pyrazole, an inhibitor of ethanol metabolism. Moreover, the earliest detectable ROS production, which was observed as soon as $15 \mathrm{~min}$, preceded the increase in membrane fluidity detected only at $30 \mathrm{~min}$ and was inhibited by 4-methyl-pyrazole but not by GM1 or UDCA, suggesting that the early ROS formation was only related to ethanol metabolism. Thus, the proposed sequence of events linking ethanol metabolism to its membrane fluidizing effect is shown in Fig. 7. Although not directly addressed in this study, the possible molecular mechanism whereby oxidative stress influences membrane fluidity of rat hepatocytes can be discussed. Lipid peroxidation by-products have been widely recognized to increase membrane fluidity either by interacting with membrane proteins (Buko et al., 1996; Subramaniam et al., 1997) or more directly by their own rearrangement (Jain et al., 1994; Gabbita et al., 1998). ROS are also able, by oxidizing tubulin, to disrupt the microtubule cytoskeleton and thereby to increase membrane fluidity (Yoon et al., 1998; RemyKristensen et al., 2000). This remains to be tested under our conditions.

As stated above, the major finding of this study is the demonstration that an early increase in membrane fluidity can be crucially involved in the enhancement of oxidative stress. Indeed, UDCA and GM1 totally inhibited ethanolinduced ROS production, lipid peroxidation, and apoptosis in rat hepatocytes, whereas Tween 20 and $\mathrm{A}_{2} \mathrm{C}$ enhanced them. The beneficial effects of UDCA and GM1 were found by EPR to be concomitant with a stabilizing effect on hepatocyte membranes, whereas Tween 20 enhanced the fluidizing effect of ethanol. It should be noted that UDCA and GM1 displayed a protection toward ethanol-induced ROS production only when ROS were evaluated after 1 or $5 \mathrm{~h}$ of incuba- 


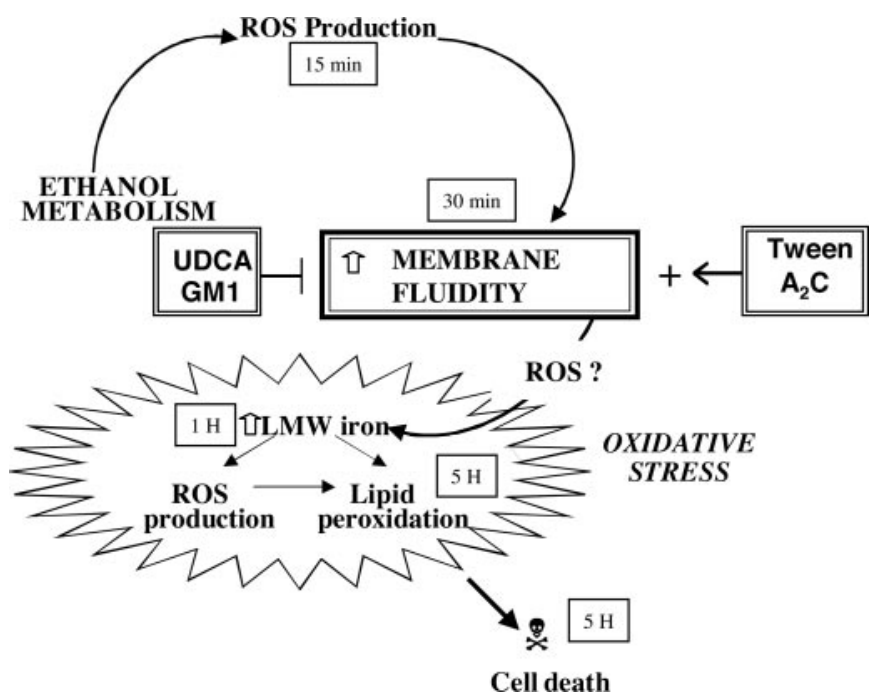

Fig. 7. Proposed model for the role of early ROS-induced fluidizing effect of ethanol in the amplification of oxidative stress. In hepatocytes, ethanol metabolism can very rapidly lead to ROS production, which in turn increased membrane fluidity. This increase can promote the elevation of low molecular weight (LMW) iron content and therefore the enhancement of ROS production and lipid peroxidation to finally trigger cell death. Membrane fluidizers (Tween or $\mathrm{A}_{2} \mathrm{C}$ ) by potentiating the increase in membrane fluidity enhanced oxidative stress, whereas membrane stabilizing agents (UDCA or GM1), by protecting from the fluidizing effect of ethanol, inhibited it.

tion with ethanol. At $15 \mathrm{~min}$, no protection was afforded by membrane stabilizing agents, unlike the inhibitor of ethanol metabolism, 4-methyl-pyrazole. This has led us to postulate a sequence of events whereby the early ROS formation was mainly due to ethanol metabolism and the late phase might also be attributed to the increase in membrane fluidity (Fig. 7). To reinforce the determinant role for the increased membrane fluidity in the initiation of oxidative stress, we have further sought the mechanism whereby membrane fluidity can elicit oxidative stress. Using EPR determination with intact hepatocytes, we show that similarly to their effects on ethanol-induced ROS production and lipid peroxidation, UDCA and GM1 inhibited the increase in low molecular weight iron content induced by ethanol, whereas Tween 20 enhanced it. It should be noted that, in previous studies (Sergent et al., 1995), the increase in low molecular weight iron was shown to be detectable only after 30 min of incubation with ethanol. Together, these results strongly support the idea that oxidative injury due to the elevation of membrane fluidity was mediated by an increase in low molecular weight iron content. Indeed, low molecular weight iron consists of iron species that can trigger oxidative stress by catalyzing the formation of a highly reactive free radical, the hydroxyl radical. In our study, desferrioxamine, a low molecular weight iron chelator, was thus found to inhibit the ROS formation induced by ethanol in rat hepatocytes. These results agree with those of Rouach et al. (1994), who observed an early increase of hepatic iron uptake from transferrin related to ROS produced by ethanol, but without establishing a link with the membrane fluidizing effect of ethanol. In this regard, it should be emphasized that an increased transferrin receptor mobility can favor its internalization (Paccaud et al., 1993) and that the major pool of low molecular weight iron is located in lysosomes or late acidic endosomes (Yu et al., 2003). In our model, it cannot be excluded that the elevation of low molecular weight iron content could be due to an increase ROS formation. Indeed, superoxide anion is well described to release iron from ferritin (Biemond et al., 1988) and oxidative stress can promote rupture of late acidic endosomes or lysosomes, thus liberating iron from these organelles (Yu et al., 2003). In this regard, our results on acridine orange-stained hepatocytes that suggested lysosome disruption would tend to support that point.

It should be kept in mind that the toxic effect of an increase in membrane fluidity, described in this study, concerns bulk membranes. Indeed, a decrease rather than an increase in fluidity has been demonstrated in inner membranes of mitochondria isolated from HepG2 cells treated with acetaldehyde, a product of ethanol metabolism (Lluis et al., 2003). Moreover, the addition of a membrane fluidizer such as $\mathrm{A}_{2} \mathrm{C}$ to these mitochondria can restore levels of glutathione, an important antioxidant for cells (Lluis et al., 2003). In this context, the use of membrane fluidizers should be done with caution, since from our results, it seems that they can be injurious for hepatocytes. On the contrary, UDCA and its conjugates seem to be good candidates for a potential therapeutic use: due to their membrane stabilizing properties, they reestablish the normality in membrane fluidity for every type of membrane. Thus, in case of ethanol intoxication, they are able to prevent both the increase of bulk membrane fluidity, as we observed it, and the decrease in the mitochondrial membrane of hepatocytes from ethanol-fed rats, thereby restoring mitochondrial glutathione levels (Colell et al., 2001). Moreover, our results about the cytoprotection afforded by UDCA and GM1 agree with previous studies that showed the prevention by UDCA of ethanol-induced necrosis and apoptosis, respectively, in human Hep G2 cells (Neuman et al., 1995) and primary rat hepatocytes (Rodrigues et al., 1998) or by GM1 of ethanol-induced death in mice neural crest cells (Chen et al., 1996) and rat cerebellar granule neurons (Saito et al., 1999). UDCA was also found to prevent lipid peroxidation in the liver from chronic ethanol-fed rats (Montet et al., 2002). But none of these studies gave a clear understanding of the mechanism involved in the protection against oxidative injury. In our study, UDCA and GM1 actions, at the doses used in our experiments, cannot be attributed to direct antioxidant properties of these compounds since they were unable to scavenge superoxide anion and hydroxyl radical in vitro and to inhibit iron-induced lipid peroxidation of rat hepatocytes. This new mechanistic demonstration of a beneficial effect of UDCA is particularly interesting because UDCA is a therapeutically relevant bile acid, already used for preventing human chronic cholestatic liver diseases (Colombo et al., 1992; Poupon et al., 2003) that might be useful in the very early stages of the alcoholic liver diseases as well.

In conclusion, the present study shows that ethanol metabolism, by triggering an early ROS production, promotes a rapid increase in membrane fluidity. The fluidizing effect of ethanol is then responsible for a secondary elevation of low molecular weight iron leading to an enhancement of ROS production that can then induce lipid peroxidation and apoptosis (Fig. 7). Consequently, early ethanol toxicity can be strongly enhanced by an increase in membrane fluidity due to ethanol metabolism itself or membrane fluidizers. The present findings clearly highlight the pivotal role of membrane fluidity in the early stages of alcoholic liver disease, 
and, in this context, membrane stabilization might be a very effective strategy to protect hepatocytes from oxidative damage.

\section{Acknowledgments}

We thank André Guillouzo for helpful discussions and critical review of the manuscript and Morgane Gorria for technical assistance.

\section{References}

Albano E (2002) Free radical mechanisms in immune reactions associated with alcoholic liver disease. Free Radic Biol Med 32:110-114.

Antras-Ferry J, Mahéo K, Morel F, Guillouzo A, Cillard P, and Cillard J (1997) Dexamethasone differently modulated TNF- $\alpha$ - and IL- $1 \beta$-induced transcription of the hepatic Mn-superoxide dismutase gene. FEBS Lett 403:100-104.

Bailey SM and Cunningham CC (1998) Acute and chronic ethanol increases reactive oxygen species generation and decreases viability in fresh, isolated rat hepatocytes. Hepatology 28:1318-1326.

Bailey SM and Cunningham CC (2002) Contribution of mitochondria to oxidative stress associated with alcoholic liver disease. Free Radic Biol Med 32:11-16.

Benedetti A, Tangorra A, Svegliati Baroni G, Ferretti G, Marucci L, Jezequel A-M, and Orlandi F (1994) Plasma membrane order parameter in periportal and perivenular hepatocytes isolated from ethanol-treated rats. Am J Physiol 266:G282G291.

Biemond P, Swaak AJG, Van Eijk HG, and Koster JF (1988) Superoxide dependent iron release from ferritin in inflammatory diseases. Free Radic Biol Med 4:185198.

Buko V, Artsukevich A, Zavodnik I, Maltsev A, Suhko L, Zimmermann T, and Dianzani MU (1996) Interactions of malondialdehyde and 4-hydroxynonenal with rat liver plasma membranes and their effect on binding of prostaglandin E2 by specific receptors. Free Radic Res 25:415-420.

Chen SY, Yang Y, Jacobson K, and Sulik K (1996) The membrane disordering effect of ethanol on neural crest cells in vitro and the protective role of GM1 ganglioside. Alcohol 13:589-595.

Colell A, Coll O, Garcia-Ruiz C, Paris R, Tiribelli C, Kaplowitz N, and FernandezCheca JC (2001) Tauroursodeoxycholic acid protects hepatocytes from ethanol-fed rats against tumor necrosis factor-induced cell death by replenishing mitochondrial glutathione. Hepatology 34:964-971.

Colombo C, Crosignani A, Assaisso M, Batazzati PM, Podda M, Giunta A, ZimmerNechemias L, and Sechell KDR (1992) Ursodeoxycholic acid therapy in cystic fibrosis-associated liver disease: a dose-response study. Hepatology 16:924-930.

Gabbita SP, Subramaniam R, Allouch F, Carney JM, and Butterfield DA (1998) Effects of mitochondrial respiratory stimulation on membrane lipids and proteins: an electron paramagnetic resonance investigation. Biochim Biophys Acta 1372 $163-173$.

Guguen-Guillouzo C, Clement B, Baffet G, Beaumont C, Morel-Chany E, Glaise D, and Guillouzo A (1983) Maintenance and reversibility of active albumin secretion by adult rat hepatocytes co-cultured with another liver epithelial cell type. Exp Cell Res 143:47-54.

Gutierrez-Ruiz MC, Gomez JL, Souza V, and Bucio L (1995) Chronic and acute ethanol treatment modifies fluidity and composition in plasma membranes of a human hepatic cell line (WRL-68). Cell Biol Toxicol 11:69-78.

Hempel SL, Buettner GR, O'Malley YQ, Wessels DA, and Flaherty DM (1999) Dihydrofluorescein diacetate is superior for detecting intracellular oxidants: comparison with 2', 7'-dichlorodihydrofluorescein diacetate, 5(and 6)-carboxy-2',7' dichlorodihydrofluorescein diacetate and dihydrorhodamine 123. Free Radic Biol Med 27:146-159.

Jain S, Thomas M, Kumar P, and Laloraya M (1994) Appearance of homogeneous smectic multilamellar microenvironments in biomembranes undergoing superoxide-initiated lipid peroxidation: lipid-dienyl radical accumulation and fluidity management in lipid bilayers. Biochem Mol Biol Int 33:853-862.

Kurose I, Higuchi H, Miura S, Saito H, Watanabe N, Hokari R, Hirokawa M, Takaishi M, Zeki S, Nakamura T, et al. (1997) Oxidative stress-mediated apoptosis of hepatocytes exposed to acute ethanol intoxication. Hepatology 25:368-378.

Lluis JM, Colell A, Garcia-Ruiz C, Kaplowitz N, and Fernandez-Checa JC (2003) Acetaldehyde impairs mitochondrial glutathione transport in HepG2 cells through endoplasmic reticulum stress. Gastroenterology 124:708-724.

Minama JB, Gomez-Cambronero LG, Lloret A, Pallardo FV, Del Omo J, Escudero A,
Rodrigo JM, Pellin A, Vina JR, Vina J, et al. (2002) Mitochondrial oxidative stress and CD95 ligand: a dual mechanism for hepatocyte apoptosis in chronic alcoholism. Hepatology 35:1205-1214.

Montet AM, Oliva L, Beaugé F, and Montet J-C (2002) Bile salts modulate chronic ethanol-induced hepatotoxicity. Alcohol Alcoholism 37:25-29.

Morel I, Lescoat G, Cillard J, Pasdeloup N, Brissot P, and Cillard P (1990) Kinetic evaluation of free malondialdehyde and enzyme leakage as indices of iron damage in rat hepatocyte cultures. Involvement of free radicals. Biochem Pharmacol 39:1647-1655.

Neuman MG, Cameron RG, Shear NH, Bellantani S, and Tiribelli C (1995) Effect of tauroursodeoxycholic and ursodeoxycholic acid on ethanol-induced cell injuries in the human Hep G2 cell line. Gastroenterology 109:555-563.

Nieto N, Friedman SL, and Cederbaum AI (2002) Stimulation and proliferation of primary rat hepatic stellate cells by cytochrome $\mathrm{P} 450$ 2E1-derived reactive oxygen species. Hepatology 35:62-73.

Nordmann R, Ribiere C, and Rouach H (1992) Implication of free radical mechanisms in ethanol-induced cellular injury. Free Radic Biol Med 12:219-240.

Ogura R, Sugiyama M, Sakanashi T, and Ninomiya Y (1988) ESR spin-labelling method of determining membrane fluidity in biological materials. Tissue culture cells, cardiac mitochondria, erythrocytes and epidermal cells. Kurume Med $J$ 35:171-182

Paccaud J-P, Reith W, Johansson B, Magnusson K-E, Mach B, and Carpentier J-L (1993) Clathrin-coated pit-mediated receptor internalization. J Biol Chem 268: 23191-23196.

Polokoff MA, Simon TJ, Harris RA, Simon FR, and Iwahashi M (1985) Chronic ethanol increases liver plasma membrane fluidity. Biochemistry 24:3114-31120.

Poupon RE, Lindor KD, Pares A, Chazouilleres O, Poupon R, and Heathcote EJ (2003) Combined analysis of the effect of treatment with ursodeoxycholic acid on histologic progression in primary biliary cirrhosis. J Hepatol 39:12-16.

Remy-Kristensen A, Duportail G, Coupin G, and Kuhry JG (2000) The influence of microtubule integrity on plasma membrane fluidity in L929 cells. Mol Membr Biol 17:95-100.

Rodrigues CMP, Guangsheng F, Ma X, Kren BT, and Steer CJ (1998) A novel role for ursodeoxycholic acid in inhibiting apoptosis by modulating mitochondrial membrane perturbation. J Clin Investig 101:2790-2799.

Rottenberg H (1991) Liver cell membrane adaptation to chronic alcohol consumption, in Drug and Alcohol Abuse Reviews, Vol. 2: Liver Pathology and Alcohol (Watson RR ed) pp 91-115, Humana Press, Totowa, NJ

Rouach H, Houze P, Gentil M, Orfanelli M-T, and Nordmann R (1994) Effects of acute ethanol administration on the uptake of $59 \mathrm{Fe}$-labeled transferrin by rat liver and cerebellum. Biochem Pharmacol 47:1835-1841.

Saito M, Saito M, Berg MJ, Guidotti A, and Marks N (1999) Gangliosides attenuate ethanol-induced apoptosis in rat cerebellar granule neurons. Neurochem Res $\mathbf{2 4}$ 1107-1115.

Sergent O, Anger J-P, Lescoat G, Pasdeloup N, Cillard P, and Cillard J (1997a) EPR determination of low molecular weight iron content applied to whole rat hepatocytes. Cell Mol Biol (Noisy-le-grand) 43:793-800.

Sergent O, Griffon B, Morel I, Chevanne M, Dubos M-P, Cillard P, and Cillard J (1997b) Effect of nitric oxide on iron-mediated oxidative stress in primary rat hepatocyte cultures. Hepatology 25:122-127.

Sergent O, Morel I, Chevanne M, Cillard P, and Cillard J (1995) Oxidative stress induced by ethanol in rat hepatocyte cultures. Biochem Mol Biol Int 35:575-583.

Subramaniam R, Roediger F, Jordan B, Mattson MP, Keller JN, Waeg G, and Butterfield DA (1997) The lipid peroxidation product, 4-hydroxy-2-trans-nonenal, alters the conformation of cortical synaptosomal membrane proteins. J Neurochem 69:1161-1169.

Yamada S and Lieber CS (1984) Decrease in microviscosity and cholesterol content of rat liver plasma membranes after chronic ethanol feeding. $J$ Clin Investig 74:2285-2289.

Yoon Y, Török N, Krueger E, Oswald B, and McNiven MA (1998) Ethanol-induced alterations of the microtubule cytoskeleton in hepatocytes. Am J Physiol 274: G757-G766.

Yu Z, Persson HL, Eaton JW, and Brunk UT (2003) Intralysosomal iron: a major determinant of oxidant-induced cell death. Free Radic Biol Med 34:1243-1252.

Zhao M, Eaton JW, and Brunk UT (2000) Protection against oxidant-mediated lysosomal rupture: a new anti-apoptotic activity of Bcl-2 ? FEBS Lett 485:104-108.

Address correspondence to: Dr. Odile Sergent, Laboratoire de Biologie Cellulaire et Végétale, Faculté de Pharmacie, 2, av. Pr. Léon Bernard, 35043 Rennes Cedex, France. E-mail: osergent@univ-rennes1.fr 